$10.1117 / 2.1200609 .0389$

\title{
Perceptual strengths and weaknesses of a 3D display
}

\author{
Christoph Hoffmann, Voicu Popescu, and Zygmunt Pizlo
}

Experiments suggest better comprehension of surface curves using a true $3 D$ display as compared with traditional flat panels.

Almost all displays today are 2D with pixels arranged in a grid. These displays render images and 3D scenes with high resolution, brightness, and color variety, but lack satisfactory support for spatial perception, even when stereo enhancements are used. There are only a few true 3D displays on the market. We investigated the strengths and weaknesses of one of these for displaying 3D images.

The Perspecta by Actuality Systems ${ }^{1}$ creates a volumetric image with the pixels arranged using cylindrical coordinates, that is, the volume is sliced by a set of radial planes. A rotating, semitransparent screen under a glass enclosure displays these radial slices according to the screen position, and the eye of the observer then synthesizes a true, volumetric image. The image can be observed from all sides, except from below.

When we acquired this 3D display in 2000, it was a secondgeneration device. It displays a 10 -inch-diameter image, using almost 200 radial slices. Each slice has a resolution of 768 by 768 pixels. Each of the approximately 100 million volume pixelsvoxels - can be displayed in one of 64 colors. In several experiments, we investigated the question of how effective this $3 \mathrm{D}$ display might be in supporting the perception of spatial relations and of curved surfaces rendered with lines. We found that the greatest strength of this kind of display is that the viewer can study a fixed 3D image from different directions, simply by walking around it.

Investigating the perception of spatial relations ${ }^{2}$ was done by presenting a sequence of 3D images of familiar objects, such as cars, buildings, and furniture. Some of the objects had rotational symmetry, others were symmetric about a mid-plane. These images were displayed in random orientation and either distorted or undistorted. A distorted image was one that was randomly stretched or compressed in such a way that the symmetry of the object was destroyed. Additional scaling and stretching was randomly applied but was not considered to distort the image as long as it preserved symmetry.
Each subject was asked to identify which object was distorted and which one was not. Images were displayed for a total of $15 \mathrm{~s}$. The performance of subjects was considered in each of the following five viewing conditions: walking around the display, fixed location at distances of $1 \mathrm{~m}$ and $2 \mathrm{~m}$, and one eye shut from a $1 \mathrm{~m}$ distance. In addition, the experiment was also conducted on a high-resolution LCD monitor (an IBM T-221 display), again monoscopically viewing the image.

We found that monocular viewing of the LCD screen was not much inferior to binocular viewing of the Perspecta from a $2 \mathrm{~m}$ distance. We believe that the explanation for this finding is that our Perspecta has relatively low contrast. Moreover, since the device displays voxels three-dimensional space, the $3 \mathrm{D}$ image is transparent, without the possibility of occlusion.

We also considered the effectiveness of comprehending the shape of curved surfaces displayed on the Perspecta as a set of lines drawn on the surface. ${ }^{3}$ Traditionally, when presented in this way, the curves are obtained by intersecting with a family of parallel planes aligned with the axes of the Euclidean coordinate system and the surface has been oriented so that the cutting planes respect at least some of the surface symmetry. We wanted to know whether the surface could be understood equally well when the curves were from cut planes that were oblique with respect to the coordinate system and did not respect surface symmetry, and even when the curves were obtained by cutting the surface with a family of spheres. In this experiment we found that the 3D display led to significantly better performance than the LCD display for all kinds of surface curves.

Volumetric 3D displays are still an emerging technology. Some of the advantages of the Perspecta include the ability to study a 3D image by examining it from different sides in the natural way. Moreover, we found that displaying space curves on the Perspecta is perceptually superior to displaying them on flat-panel displays. Disadvantages of our volumetric display include low contrast and a slightly wobbly image, which we believe to be a mechanical issue of the drive mechanism. Advances in technology should ameliorate some of these disadvantages, although

Continued on next page 
the inability to display opaque surfaces is inherent in this particular technology.

\section{Author Information}

\section{Christoph Hoffmann, Voicu Popescu}

Computer Science

Purdue University

West Lafayette, IN

Christoph Hoffmann does research in geometric computing, geometric constraint solving, visualization, and applications in simulation and design. He is co-director of Purdue's Computing Research Institute (CRI) and of Purdue's Product Lifecycle Management Center of Excellence (PLM). In addition, Hoffmann has contributed papers to SPIE conferences.

Voicu Popescu is an assistant professor of computer science at Purdue University. His research interests span computer graphics, visualization, and computer vision. In addition, he occasionally contributes to SPIE conferences.

\section{Zygmunt Pizlo}

Dept of Psychology

Purdue University

West Lafayette, IN

Zygmunt Pizlo is a professor of Psychological Sciences at Purdue University. His research interests include computational modeling of human vision, motor control, and cognition. In addition, he has been a member of the program committee for conferences in SPIE's Symposium on Electronic Imaging.

\section{References}

1. Actuality Systems, Perspecta Display. www.actualitysystems.com

2. P. Rosen, Z. Pizlo, C. Hoffmann, and V. Popescu, Perception of 3D Spatial Relations for 3D Displays, Proc. SPIE 5291, 2004.

3. C. Hoffmann, Z. Pizlo, V. Popescu, and S. Price, Perception of Surfaces from Line Drawings, to appear., 2006. http:/ / www.cs.purdue.edu/cgvlab/publications.html 